\author{
Atividade Antichagásica de Lignanas e Neolignanas \\ Souza, V. A.; Nakamura, C. V.; Corrêa, A. G.* \\ Rev. Virtual Quim., 2012, 4 (3), 197-207. Data de publicação na Web: 28 de abril de 2012 \\ http://www.uff.br/rvq
}

\title{
Antichagasic Activity of Lignans and Neolignans
}

Abstract: Lignans and neolignans are a large group of natural products characterized by the coupling of two units of phenylpropanoids. Several reports in the literature have been described the synthesis of lignans and neolignanas due to their interesting pharmacological profile. In this review we discuss the biological activity of natural or synthetic lignans and neolignans towards the parasite Trypanosoma cruzi, which causes the Chagas' disease.

Keywords: Lignans; neolignans; natural products; Chagas' disease; Trypanosoma cruzi.

\section{Resumo}

As lignanas e neolignanas compreendem uma classe de produtos naturais com uma grande diversidade de estruturas químicas, sendo formadas pelo acoplamento de duas unidades fenilpropanoides. Vários trabalhos têm sido descritos na literatura relatando a síntese de lignanas e neolignanas. Este crescente interesse se deve a grande diversidade de atividades biológicas que esta classe de compostos vem demonstrando. Nesta revisão serão abordados os trabalhos que descrevem à atividade de lignanas e neolignanas naturais e sintéticas contra o parasita Trypanosoma cruzi, o causador da doença de Chagas.

Palavras-chave: Lignanas; neolignanas; produtos naturais; doença de Chagas; Trypanosoma cruzi.

\footnotetext{
* Universidade Federal de São Carlos, Departamento de Química, CEP 13565-905, São Carlos, São Paulo, Brasil.

Magcorrea@ufscar.br

DOI: $10.5935 / 1984-6835.20120017$
}

Rev. Virtual Quim. |Vol 4| |No. 3| |197-207| 


\title{
Atividade Antichagásica de Lignanas e Neolignanas
}

\section{Vanessa A. de Souza, ${ }^{a}$ Celso V. Nakamura, ${ }^{b}$ Arlene G. Corrêa ${ }^{a, *}$}

\author{
aUniversidade Federal de São Carlos, Departamento de Química, CEP 13565-905, São Carlos, São Paulo, Brasil. \\ bUniversidade Estadual de Maringá, Departamento de Ciências Básicas da Saúde, Laboratório de Inovação \\ Tecnológica no Desenvolvimento de Fármacos e Cosméticos, Av. Colombo 5790, CEP 87020-900 Maringá, \\ Paraná, Brasil. \\ *agcorrea@ufscar.br
}

Recebido em 2 de fevereiro de 2012. Aceito para publicação em 27 de abril de 2012

\section{Introdução}

2. Quimioterapia da doença de Chagas

3. Lignanas, neolignas e análogos ativos contra formas do T. cruzi

4. Conclusões

\section{Introdução}

As lignanas e neolignanas compreendem uma classe de produtos naturais com uma grande diversidade de estruturas químicas e atividades farmacológicas, sendo formadas pelo acoplamento de duas unidades fenilpropanoides (eugenol, álcool coniferílico, isoeugenol, etc.). A forma como estas unidades são ligadas entre si determinam sua classificação: as lignanas ligam-se pela posição 8 e $8^{\prime}$ da cadeia alifática, enquanto que as neolignanas são geradas pela ligação de qualquer outra posição da unidade fenilpropanoide que não seja 8 e 8 ' (Figura 1). ${ }^{1}$ As oxineolignanas (geradas das ligações $\mathrm{C}-\mathrm{O}-\mathrm{C}^{\prime}$ ) apresentam uma variedade de atividades biológicas, como antifúngica, leishmanicida, antioxidante, esquistossomicida, etc. ${ }^{2-5}$

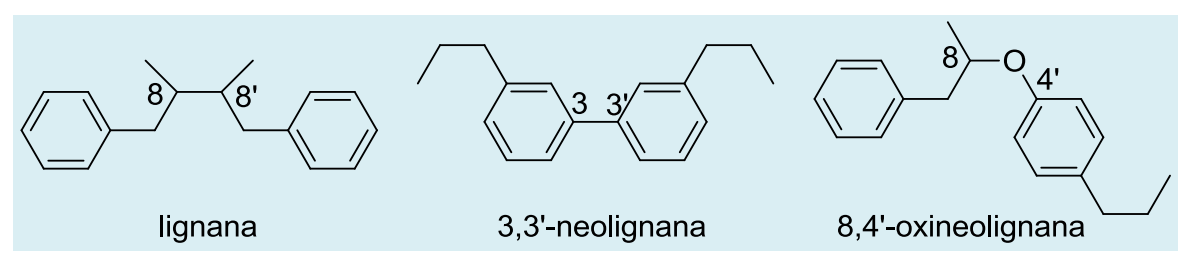

Figura 1. Esqueletos carbônicos de lignanas e neolignanas ${ }^{1}$

O Prof. Otto Gottlieb dedicou-se ao estudo desta classe de compostos, que apesar de conhecida de longa data, começou a ter suas propriedades biológicas reconhecidas a partir de seus trabalhos. ${ }^{6}$ Lignoides e oligômeros de arilpropanoides possuem atividade aleloquímica, principalmente nas plantas possuidoras de propriedades farmacológicas. As lignanas são acumuladas em madeiras como resposta a ferimentos mecânicos ou a invasão de fungos ou bactérias. No nosso organismo elas apresentam também funções importantes, como o secoisolariciresinol e matairesinol (Figura 2), que estão presentes em várias plantas, incluindo Forsythia intermedia, feijão e centeio. Durante a digestão, as bactérias intestinais convertem o secoisolariciresinol (1) e o matairesinol (2) em enterodiol (3) e 
enterolactona (4), respectivamente. Estas lignanas sofrem circulação entero-hepática, sendo conjugadas no fígado e excretadas na bile, desconjugadas no intestino por enzimas bacterianas, absorvidas através da mucosa intestinal, retornando ao fígado pela circulação. Acredita-se que o secoisolariciresinol (1) e matairesinol (2) são responsáveis por reduzir a taxa de incidência de câncer de próstata e de mama, através de uma alimentação rica em grãos e vegetais que contém altas concentrações de $\mathbf{1}$ e $\mathbf{2}^{7}$

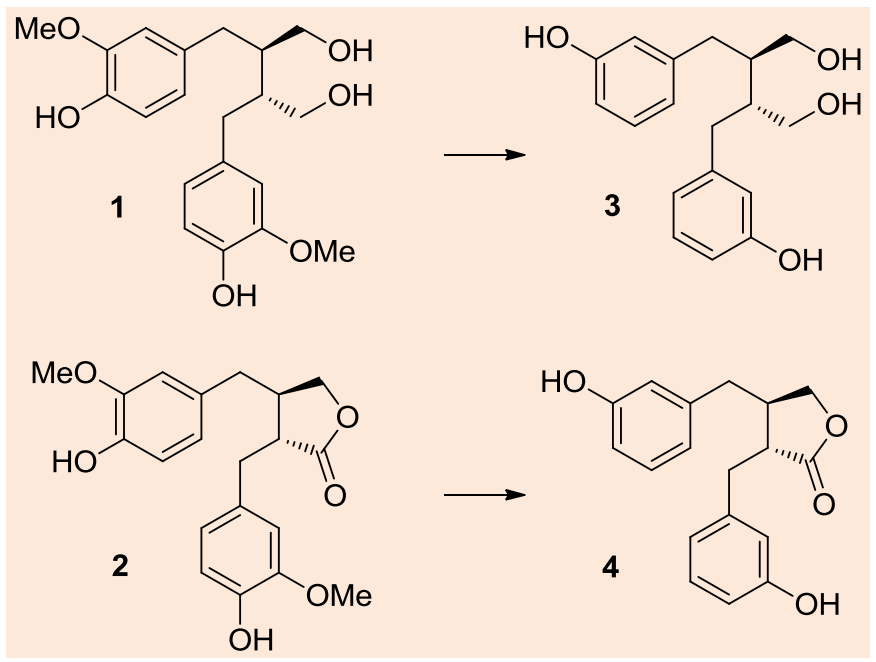

Figura 2. Secoisolariciresinol e matairesinol e sua conversão no organismo humano

Outro exemplo importante desta classe de compostos é o (-)-ácido plicático (5), um dos principais constituintes do cedro vermelho, que contribui substancialmente para sua cor, qualidade e durabilidade, permitindo que a espécie sobreviva por mais de 3000 anos. O teniposídeo (6) e etoposídeo
(7), derivados semi-sintéticos da (-)-podofilotoxina (8), são usados em tratamentos de certos cânceres, e a (+)-sesamina (9) encontrada em sementes de gergelim, apresenta propriedades antioxidantes in vitro (Figura 3$)^{7}$<smiles></smiles>

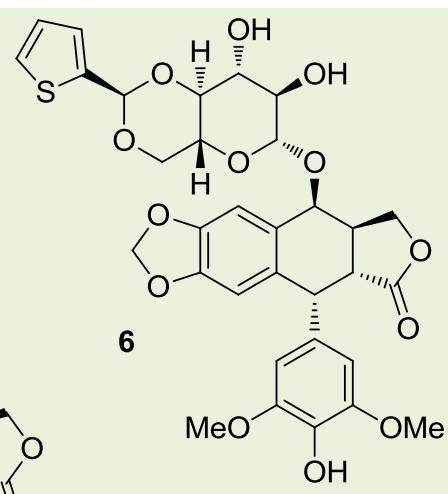

7<smiles>CO[C@H]1O[C@@H]2CO[C@H](C)O[C@H]2[C@H](O)[C@H]1O</smiles><smiles>C[C@@H]1c2cc3c(cc2[C@H](O)[C@@H]2COC(=O)[C@H]12)OCO3</smiles>

Figura 3. Lignanas ariltetralínicas e di-hidrofuranos que apresentam atividades biológicas importantes

Vários trabalhos foram descritos na literatura relatando a síntese de lignanas e neolignanas. Este 
crescente interesse se deve a grande diversidade de atividades biológicas que esta classe de compostos vem mostrando, como atividade contra leishmaniose, malária, doença de Chagas, anti-PAF, antitumoral, inseticida, atividade antimicrobiana, anti-inflamatória e analgésica. ${ }^{8-13}$

\section{Quimioterapia da doença de Chagas}

A doença de Chagas ou tripanossomíase americana é causada pelo protozoário flagelado Trypanosoma cruzi, que é transmitido ao hospedeiro humano, principalmente pelo vetor hematófago conhecido como "barbeiro" (Triatoma infestans, Panstrongylus megistus, entre outras). Esta doença afeta cerca de 10 milhões de pessoas do sul dos Estados Unidos até a Patagônia, causando 10.000 mortes por ano, sendo que outros 25 milhões de indivíduos vivem em áreas de risco de contaminação. ${ }^{14}$

A doença de Chagas foi descoberta, em 1909, pelo médico brasileiro Carlos Chagas. Embora todo o ciclo da doença tenha sido descrito entre 1909 a 1935, não há ainda uma estratégia quimioterapêutica efetiva para o seu tratamento, que vem sendo realizado de forma muito mais sintomática que etiológica. Os tratamentos clínicos empregados na década de 60-70 foram com o benzonidazol (10) (Rochagan, Roche) e nifurtimox (11) (Lampit, Bayer - não se encontra disponível comercialmente no Brasil), porém estes fármacos apresentam vários efeitos colaterais, como: anorexia, náuseas, vômitos, alergia cutânea e neuropatia periférica. O composto 10 é o único fármaco disponível para o tratamento da doença e apresenta índice de cura de $70-80 \%$ na fase aguda, e 10-20\% na fase crônica, o nifurtimox apresenta o mesmo índice de cura que o benzonidazol, no entanto apresenta efeitos citotóxicos mais pronunciados (Figura 4). ${ }^{15}$
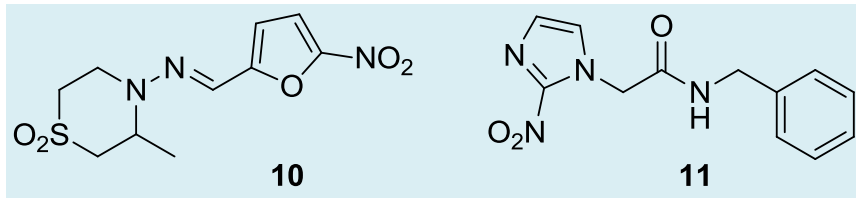

Figura 4. Benzonidazol 10 e nifurtimox 11

Apesar do número de mortes causadas por doenças infecciosas ter diminuído de $50 \%$ para $5 \%$ ao longo dos últimos oitenta anos, estas doenças continuam sendo um problema de saúde pública no Brasil. A proporção do total de mortes causadas por doenças infecciosas caiu consideravelmente, mas o número de mortes causadas pela doença de Chagas permaneceu estável. Esta doença apresenta duas fases características, uma aguda que corresponde à infecção e disseminação do protozoário no organismo, geralmente assintomática; e uma crônica que se manifesta como miocardiopatia, megaesôfago ou megacólon. A miocardiopatia é muito grave, com uma alta taxa de letalidade que pode alcançar até $80 \%$ em um período de cinco anos após o primeiro internamento, atingindo principalmente homens entre 30 e 40 anos de idade. ${ }^{16}$

Em 2006, o Ministério da Saúde recebeu a certificação internacional de eliminação da transmissão da doença de Chagas conferida pela Organização Pan-Americana da Saúde e concedida pela OPS/OMS. ${ }^{17}$ Apesar dos grandes esforços realizados para erradicar o vetor da doença, ela ainda representa um sério desafio, em razão do longo período de latência. Apesar de uma queda no número de óbitos de 5,91\%, 3,5 milhões de indivíduos ainda estão infectados. ${ }^{18} \mathrm{~A}$ grande dificuldade de eliminação do parasita se deve as diversas vias alternativas de transmissão (transfusões de sangue, transmissão mãe-bebê e mais raramente por alimentos frescos contaminados), e, também, pelo grande número de vetores e reservatórios ainda existentes.

O desenvolvimento de novos fármacos para o tratamento da doença de Chagas não é prioridade das indústrias farmacêuticas, devido ao alto custo dos investimentos e a falta de um mercado potencial e seguro nos países em desenvolvimento, pois a doença afeta principalmente setores menos favorecidos das sociedades, desassistidos pelo poder público e negligenciados, por razões econômicas. Dos 1393 fármacos introduzidos no mercado farmacêutico entre 1975 e 1999, menos de 1,1\% foram dirigidas para as doenças tropicais e para a tuberculose. ${ }^{15}$ Por isso, há necessidade de pesquisas envolvendo o desenvolvimento de novos fármacos no combate à doença de Chagas.

Um dos grandes problemas enfrentados na busca de um fármaco eficiente para o tratamento da doença de Chagas é o fato do parasita possuir um complexo 
ciclo biológico, que envolve três formas distintas: (i) epimastigota, forma presente no vetor e em cultura axênica; (ii) tripomastigota, forma sanguínea circulante e infectante; e (iii) amastigota, forma de replicação intracelular. Outro problema na identificação do protótipo está relacionado com as cepas testadas, porque algumas são mais resistentes aos fármacos comumente usados, e estas diferenças devem ser analisadas com cautela, porque podem resultar em diferentes mecanismos de ação e defesa. ${ }^{19,20}$ Alguns grupos têm utilizado mais de uma única estirpe, e essa comparação pode ser importante para melhorar a avaliação dos efeitos. Poucos grupos de pesquisa têm usado mais que uma cepa para avaliar o efeito de novos compostos, e o uso de cepas diferentes podem nos levar a informações importantes sobre a atividade dos novos fármacos.

\section{Lignanas, neolignas e análogos ativos contra formas do $T$. cruzi}

Os produtos naturais são uma fonte potencial de novos fármacos, os quais podem, no futuro, substituir muitos medicamentos atuais que apresentam baixa eficácia e efeitos colaterais. Recentemente, Izumi e col. fizeram uma revisão, onde descrevem 18 espécies de plantas que já tiveram suas frações hexânicas ou metanólicas testadas contra diferentes fases da vida do $T$. cruzi. Algumas apresentaram excelentes atividades biológicas, no entanto apenas $10 \%$ das plantas ativas tiveram seus princípios ativos isolados e caracterizados. $^{21}$ Os autores além de apresentaram uma relação de 136 compostos isolados de plantas testados, nas últimas décadas, frente as formas infectantes do T. cruzi, apresentam uma relação de estrutura-atividade destes compostos.

Dentre os compostos isolados, 13 são lignanas. A maior parte destes compostos apresentou atividade. Podem-se destacar as lignanas 12, 13 e 14 com $\mathrm{Cl}_{50}=$ 2,$2 ; 4,4 \mu \mathrm{M}$ e 3,47 $\mu \mathrm{g} / \mathrm{mL}$ respectivamente, após $24 \mathrm{~h}$ de incubação frente à forma tripomastigota; as lignanas 15 - 21 com $\mathrm{Cl}_{50}$ na faixa de 7,0 a 24,5 $\mathrm{\mu g} / \mathrm{mL}$, após $96 \mathrm{~h}$ de incubação, frente à forma epimastigota; e o composto $15 \mathrm{com} \mathrm{Cl}_{50}=5 \mu \mathrm{g} / \mathrm{mL}$, após $48 \mathrm{~h}$ de incubação, contra a forma amastigota do T. cruzi. O composto 15 apresentou baixo efeito citotóxico e foi significativamente mais ativo que o benzonidazol 10 (Figura 5). Sua ação tripanocida pode estar associada com a disfunção mitocondrial e dano oxidativo, que pode desencadear efeitos destrutivos sobre as moléculas biológicas do $T$. cruzi, levando a sua morte. $^{21-23}$

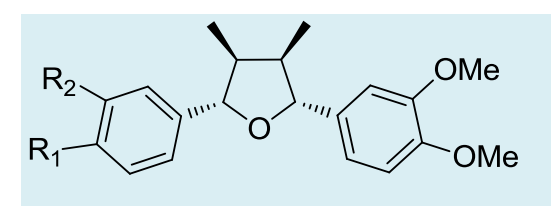

12, $\mathrm{R}_{1}$ e $\mathrm{R}_{2}=\mathrm{OCH}_{2} \mathrm{O}$<smiles>COc1cc([C@H]2O[C@@H](c3cc(OC)c4c(c3)OCO4)[C@H](C)[C@H]2C)cc2c1OCO2</smiles>

13, $R_{1}=O M e, R_{2}=O M e$

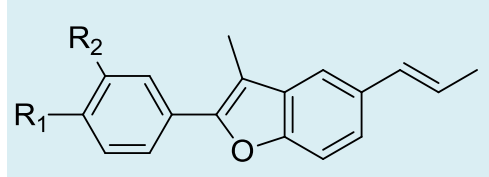

15, $\mathrm{R}_{1}=\mathrm{OH}$ e $\mathrm{R}_{2}=\mathrm{OMe}$

16, $\mathrm{R}_{1}=\mathrm{OH}$ e $\mathrm{R}_{2}=\mathrm{H}$

17, $\mathrm{R}_{1}=$ OMe e $\mathrm{R}_{2}=\mathrm{Me}$

18, $R_{1}=O M e$ e $R_{2}=H$

19, $\mathrm{R}_{1}$ e $\mathrm{R}_{2}=\mathrm{OCH}_{2} \mathrm{O}$

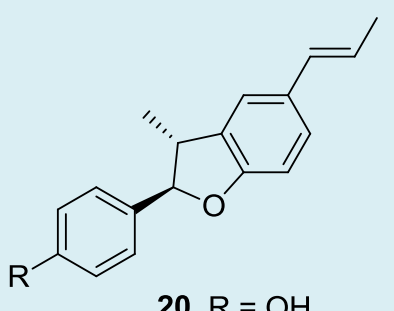

20, $\mathrm{R}=\mathrm{OH}$

21, $\mathrm{R}=\mathrm{OMe}$

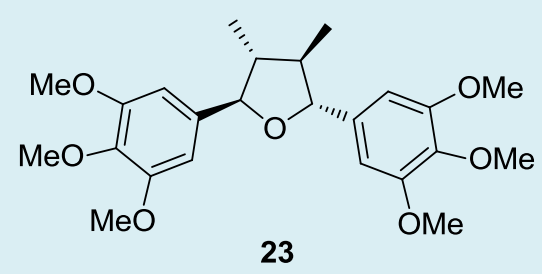

Figura 5. Lignanas com atividade antichagásica 
Felippe et al. relataram a atividade tripanocida de 5 lignanas isoladas de Peperomia blanda. Todos os compostos isolados foram caracterizados e avaliados frente às formas epimastigota de T. cruzi, incubados por um período de $72 \mathrm{~h}$. Os compostos 24-28 apresentaram $\mathrm{Cl}_{50}$ entre 9,6 a $25,4 \mathrm{\mu g} / \mathrm{mL}$, sendo a lignana 26 a mais ativa (Figura 6). ${ }^{24}$<smiles>[R]c1cc([C@H]2O[C@@H](c3cc(OC)c4c(c3)OCO4)[C@H](C)[C@H]2C)cc(OC)c1[R]</smiles>

24, $\mathrm{R}_{1}=\mathrm{OMe}, \mathrm{R}_{2}=\mathrm{OH}$

25, $\mathrm{R}_{1}=\mathrm{OMe}, \mathrm{R}_{2}=\mathrm{OMe}$

26, $\mathrm{R}_{1}=\mathrm{R}_{2}=\mathrm{OCH}_{2} \mathrm{O}$<smiles>[R]C[C@H]1[C@@H](c2cc([R2])c([R3])c(OC)c2)O[C@H](c2cc(OC)c(OC)c(OC)c2)[C@H]1C</smiles>

27, $\mathrm{R}_{1}=\mathrm{H}, \mathrm{R}_{2}=\mathrm{OMe}, \mathrm{R}_{3}=\mathrm{OH}$

28, $\mathrm{R}_{1}=\mathrm{OH}, \mathrm{R}_{2}=\mathrm{R}_{3}=\mathrm{OCH}_{2} \mathrm{O}$

Figura 6. Lignanas isoladas e testadas frente à forma epimastigota de T. cruzi

Bernardes et al. relataram a síntese de análogos dos produtos naturais veraguensina (22) e grandisina (23), que são compostos que vem apresentando potente atividade in vitro frente a forma tripomastigota, com $\mathrm{Cl}_{50}=2,3$ e $3,7 \mu \mathrm{M}$ respectivamente. A partir das arilcetonas $\alpha, \beta$ insaturadas 29 e arilaldeídos $\mathbf{3 0}$, através da reação de adição conjugada de Michael, estes autores obtiveram $\mathrm{O}$ composto $\mathbf{3 1}$ com diferentes substituintes, que foi reduzido com $\mathrm{NaBH}_{4}$ formando os compostos 32 (Esquema 1). Todos os compostos foram testados frente às formas tripomastigotas, sendo os mais ativos o 31a e 31b com $\mathrm{Cl}_{50}=100$ e 105 $\mu \mathrm{M}$ respectivamente frente à Cepa $\mathrm{Y}$ e o composto 32b com $\mathrm{Cl}_{50}=10 \mu \mathrm{M}$ (cepa Y) e $\mathrm{Cl}_{50}=200 \mu \mathrm{M}$ (cepa Bolívia). ${ }^{23}$<smiles>[R]c1cc(C(=O)C=C)cc(OC)c1OC</smiles>

29<smiles>[R]c1cc(C=O)cc([R])c1[R3]</smiles>

30

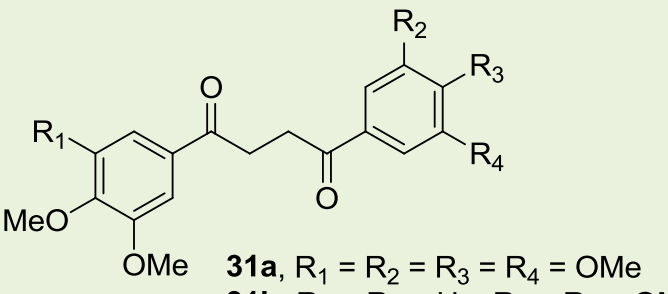

31b, $R_{1}=R_{2}=H$ e $R_{3}=R_{4}=O M e$

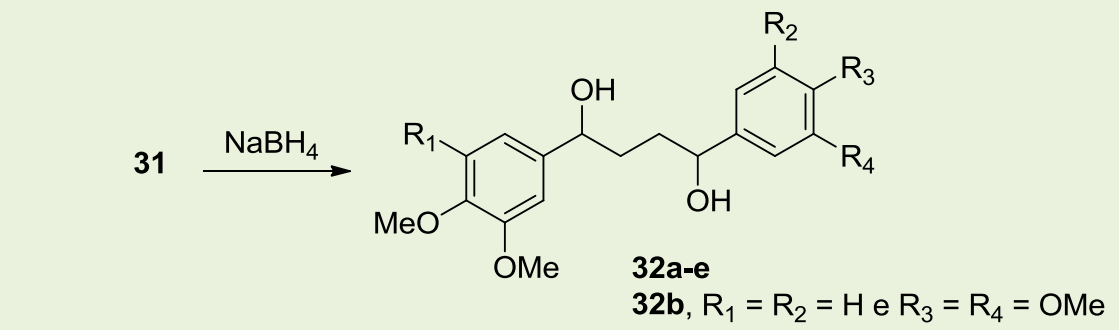

Esquema 1. Compostos análogos a lignanas (ETB = brometo de 3-etil-5-(2-hidroxietil)-4-metil tiazolila)

Souza et al. descreveram a atividade tripanocida de alguns compostos derivados da (-)-cubebina, produto natural extraído de uma espécie de pimenta indiana, a Piper cubeba. Os derivados semi-sintéticos mais ativos contra a forma amastigota do $T$. cruzi foram a (-)-hinoquinina (33), (-)-O-benzil cubebina (34) e (-)-( $N, N$-dimetilaminoetil)cubebina (35), com $\mathrm{Cl}_{50}=0,7,5,7$ e $4,7 \mu \mathrm{M}$, respectivamente, após $24 \mathrm{~h}$ de incubação. O composto $\mathbf{3 3}$ foi submetido a ensaios in vivo, e apresentou um $\mathrm{Cl}_{50}=0,67 \mu \mathrm{M}$ frente às formas epimastigotas do T. cruzi. Estudos posteriores demonstraram também que a (-)-hinoquinina, além de não ser genotóxica, é eficaz na redução de danos cromossômicos induzidos por dexorrubicina, apresentando efeito antioxidante na mitocôndria do parasita (Figura 7). ${ }^{9,25,26}$ 
<smiles>O=C1OC[C@H](Cc2ccc3c(c2)OCO3)[C@H]1Cc1ccc2c(c1)OCO2</smiles>

Figura 7. Derivados semi-sintéticos da (-)-cubebina
Silva et al. relataram a síntese do metilpluviatolido a partir do intermediário 36 , obtido de acordo com as metodologias descritas por Landais e Charlton. ${ }^{27,28} \mathrm{O}$ isômero (-)-37 foi obtido através de uma hidrogenólise de 36 , e o (+)-38 foi obtido em três etapas a partir de $\mathbf{3 6}$, por uma reação de acetilação, seguida de uma eliminação com DBU e hidrogenação. Tanto a mistura como os isômeros (-)-37 e (+)-38

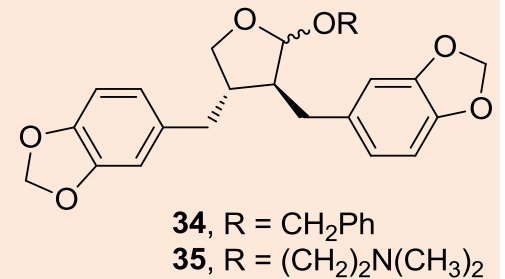

34, $\mathrm{R}=\mathrm{CH}_{2} \mathrm{Ph}$

35, $\mathrm{R}=\left(\mathrm{CH}_{2}\right)_{2} \mathrm{~N}\left(\mathrm{CH}_{3}\right)_{2}$ puros foram ensaiados frente às formas tripomastigotas do $T$. cruzi. A mistura resultou em um $\mathrm{Cl}_{50}=89,3 \mu \mathrm{M}$, e apenas (-)-37 foi ativo, apresentando $\mathrm{Cl}_{50}=18,7 \mu \mathrm{M}$. Foi testada a atividade frente à enzima gGAPDH do parasita, no entanto o composto não se mostrou ativo e nem impediu a produção de NO e peróxido nas concentrações testadas (Esquema 2). ${ }^{29}$
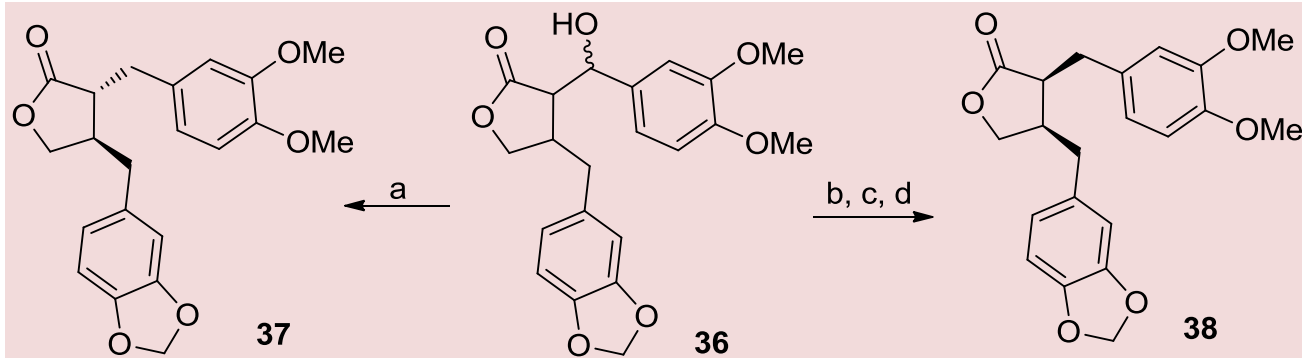

Esquema 2. a) Pd/C, 4 atm, $\mathrm{H}_{2}$, EtOH, $\mathrm{HClO}_{4}, 60$ h, t.a.; b) THF, $\left.\mathrm{Ac}_{2} \mathrm{O}, \mathrm{Et}_{3} \mathrm{~N}, \mathrm{DMAP}, 2 \mathrm{~h}, \mathrm{t} . \mathrm{a} . ; \mathrm{c}\right) \mathrm{DBU} \mathrm{CH}_{2} \mathrm{Cl}_{2}, 5 \mathrm{~h}$, t.a.; d) $\mathrm{H}_{2}, 4$ atm, Pd/C, EtOH, t.a., $4 \mathrm{~h}$

Del Omo et al. relataram a atividade anti-chagásica de uma série de estilbenos naturais e sintéticos, frente as formas epimastigota e tripomastigota de $T$. cruzi, e alguns compostos mostraram-se tão potentes quanto o benzonidazol (10). Os compostos mais ativos foram o 39 e 40 que apresentaram $\mathrm{Cl}_{50}=12$ e 2,3 $\mu \mathrm{g} / \mathrm{mL}$ frente às formas tripomastigota quando incubados por um período de $24 \mathrm{~h}$. Os compostos 40 e 41 apresentaram $\mathrm{Cl}_{50}=10$ e $20 \mu \mathrm{g} / \mathrm{mL}$, respectivamente, frente às formas epimastigota quando incubados por um período de $72 \mathrm{~h}$ (Figura $8)^{30}$

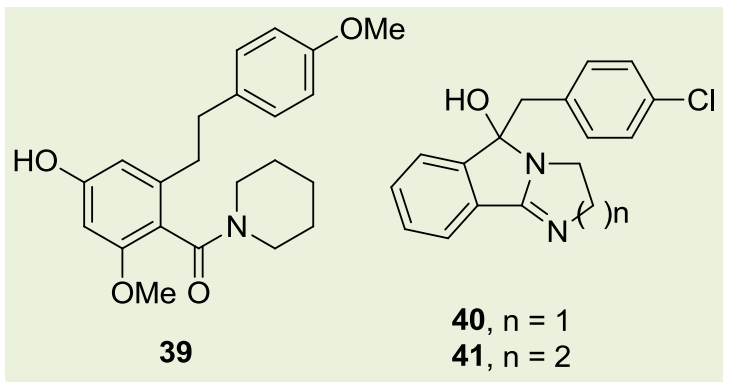

Figura 8. Estilbenos semi-sintéticos e sintéticos

Cabral et al. isolaram duas neolignanas, licarina A (42) de Nectandra glabrescens Benth e buchelina (43) de Ocotea cymbarum Kunth. Estas duas neolignanas tiveram suas atividades tripanocida avaliadas contra a forma tripomastigota (incubadas por $24 \mathrm{~h}$, utilizandose cepa $Y$ do parasita), e apresentaram $\mathrm{Cl}_{50}=960$ e 
$520 \mu \mathrm{M}$, respectivamente, e frente à forma epimastigota (incubadas por $96 \mathrm{~h}$, utilizando-se cepa
Dm 28 do parasita) com um $\mathrm{Cl}_{50}=462$ e $756 \mu \mathrm{M}$, respectivamente (Figura 9). ${ }^{31}$

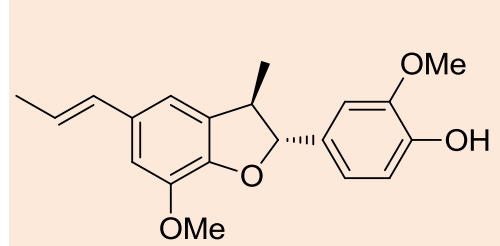

42

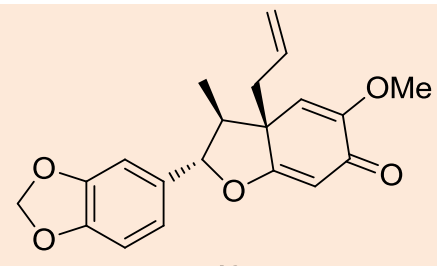

43

Figura 9. Neolignanas testadas contra a forma epimastigota de T. cruzi

Pereira et al. prepararam a licarina A (42) através do acoplamento oxidativo do isoeugenol (44) (Esquema 3). O composto 45, obtido como uma mistura racêmica foi ensaiado frente à forma tripomastigota de T. cruzi incubadas por $24 \mathrm{~h}$, apresentando $\mathrm{Cl}_{50}=127,17 \mu \mathrm{M}$. Os enantiômeros foram separados por HPLC quiral e testados, sendo que o enantiômero (-)-46 apresentou $\mathrm{Cl}_{50}=23,5 \mu \mathrm{M}$ e o enantiômero (+)-42 um $\mathrm{Cl}_{50}=87,7 \mu \mathrm{M} .^{32}$

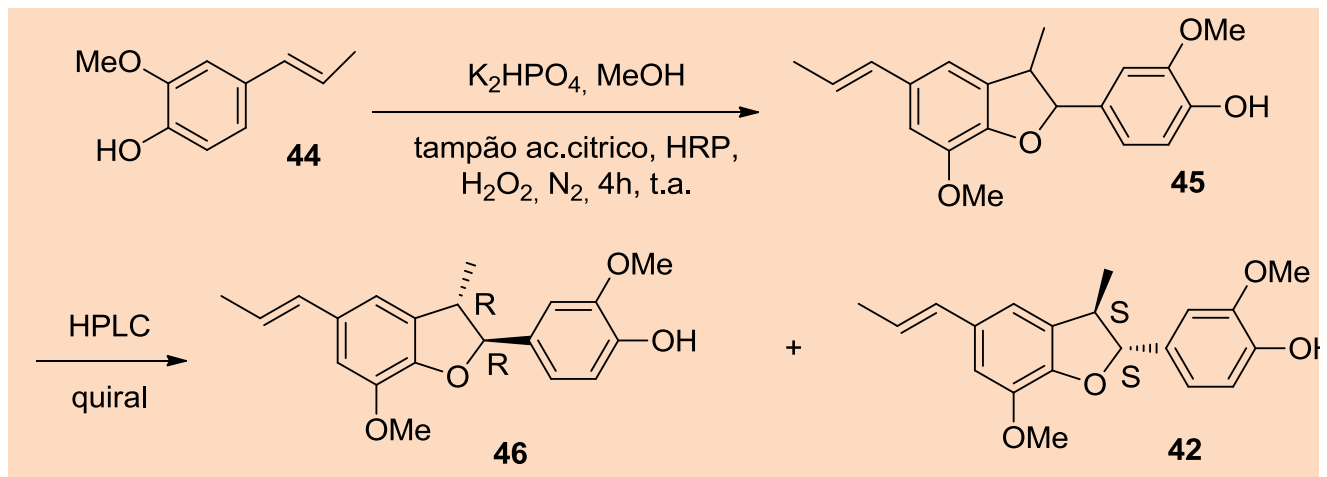

Esquema 3. Neolignanas sintetizadas

Os compostos isolados e sintetizados são na maioria das vezes ensaiados frente à forma epimastigota do $T$. cruzi, pois as formas tripomastigota e amastigota requerem mais recursos para manter modelos animais ou culturas celulares infectadas. A comparação dos resultados do ensaio biológico pode ser um pouco complicada, pois podem ocorrer algumas mudanças na metodologia empregada. Isto se deve as diferentes condições necessárias do meio de cultura para cada estágio do parasita, e diferentes meios de cultura de células do hospedeiro. Além da precisão dos dados, o tempo de incubação pode variar de $24,48,72,96 \mathrm{~h}$ e às vezes até $168 \mathrm{~h}$ em alguns estudos, assim como os tipos de cepas empregadas. ${ }^{21}$

Como mostrado acima, Cabral et al. e Pereira et al. ensaiaram a licarina A (42) empregando o mesmo tempo de incubação e a mesma cepa $Y$, no entanto os valores obtidos nos ensaios foram diferentes $\left(\mathrm{Cl}_{50}=\right.$ 960 e $87,7 \mu \mathrm{M}$, respectivamente). Esta diferença se deve às condições empregadas na realização do ensaio. Na metodologia de Cabral, é utilizada acetona para solubilizar o composto, enquanto que Pereira emprega DMSO. Há também uma variação na preparação dos meios empregados nos dois artigos, levando a esta diferença de resultados entre os dois ensaios. Devido a estes fatores há uma grande necessidade de se padronizar as condições empregadas nos ensaios biológicos em geral.

Garcia e Azambuja avaliaram os efeitos de algumas lignanas e neolignanas adicionados na dieta de Rhodnius prolixus, um dos principais vetores causadores da doença de Chagas. Os compostos buchelina (43), podofilotoxina (8), pinoresinol (47), sesamina (9), licarina $A$ (42) e o ácido nordihidroguaiaretico (NDGA) (48) (Figura 10) foram testados separadamente e em associação. A associação de $\mathbf{4 7}$ com $\mathbf{4 8}$ inibiu significativamente a quantidade do parasita, apresentando um $\mathrm{DE}_{50}<20$ $\mu \mathrm{g} / \mathrm{mL}, 43$ com 8 diminuiu a excreção do $T$. cruzi em $100 \mu \mathrm{g} / \mathrm{mL}$. O melhor resultado foi pela associação de 43 com 48 que diminuiu drasticamente a quantidade 
de parasita no trato digestivo para $10 \mu \mathrm{g} / \mathrm{mL}$. Estes resultados fazem com que compostos desta classe sejam interessantes candidatos a serem utilizados no combate à transmissão dessa doença. ${ }^{33}$

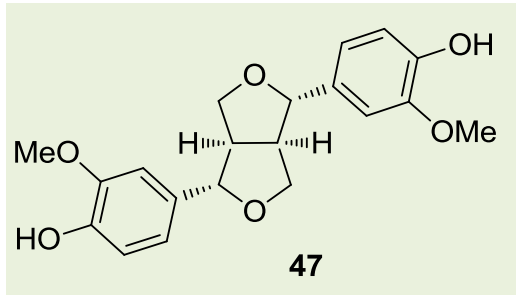

47

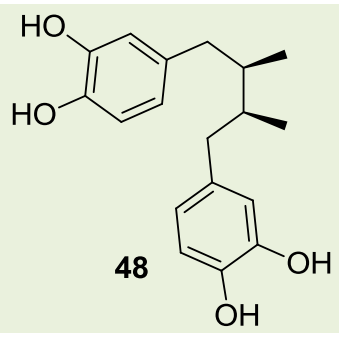

Figura 10. Estrutura de algumas lignoides inseridas na dieta de Rhodnius prolixus

Abe et al. testaram 43 tipos de extratos orgânicos de 39 plantas frente ao T. cruzi, dentre eles o melhor resultado foi com as raízes de Aristolochia taliscana, conhecida como guaco. $O$ extrato matou as formas epimastigota do $T$. cruzi em concentração de 0,5 $\mathrm{mg} / \mathrm{mL}$, em 48 h. Com o intuito de identificar os principais compostos ativos da planta, foi feito o biomonitoramento, encontrando-se 4 neolignanas, eupomatenoide-7 (15), licarina A (42), eupomatenoide-1 (49) e licarina B (50), e duas lignanas, austrobailignan-7 (51) e fragransina $E_{1}$ (52) (Figura 11). Todos os compostos tiveram sua concentração mínima para matar $100 \%$ dos parasitas $\left(\mathrm{CM}_{100}\right)$ calculadas, para as formas epimastigota incubadas por um período de $48 \mathrm{~h}$. Os compostos $49 \mathrm{e}$ 50 foram inativos. No entanto, os compostos 15, 42, 51 e 52 foram ativos, com $M_{100}=25 ; 40 ; 75$ e 50 $\mu \mathrm{g} / \mathrm{mL}$ respectivamente. ${ }^{34}$<smiles>C/C=C/c1cc(OC)c2oc(-c3ccc4c(c3)OCO4)c(C)c2c1</smiles><smiles>COc1cc([C@@H]2O[C@@H](c3ccc4c(c3)OCO4)[C@H](C)[C@H]2C)ccc1O</smiles><smiles>C/C=C/c1cc(OC)c2c(c1)[C@H](C)[C@H](c1ccc3c(c1)OCO3)O2</smiles><smiles>COc1cc([C@H]2O[C@@H](c3ccc4c(c3)OCO4)[C@H](C)[C@H]2C)ccc1O</smiles>

Figura 11. Neolignanas 49 e 50 inativas e lignanas $\mathbf{5 1}$ e $\mathbf{5 2}$ ativas frente ao T. cruzi

\section{Conclusões}

Embora todo o ciclo da doença de Chagas tenha sido descrito há mais de 100 anos, não há ainda uma estratégia quimioterapêutica efetiva para o seu tratamento, que vem sendo realizada de forma muito mais sintomática que etiológica. Em vista da necessidade de novas substâncias com atividade biológica sobre $T$. cruzi, o interesse pela pesquisa vem crescendo com o intuito de se obter compostos capazes de atuarem sobre o parasita, desprovidos de graves efeitos colaterais. Estudos desenvolvidos envolvendo lignanas e neolignanas têm demonstrado a importante atividade destes compostos sobre diferentes formas do parasita. A continuidade destes Rev. Virtual Quim. |Vol 4| |No. 3| |197-207| estudos pode contribuir para o desenvolvimento de novos fármacos para o tratamento da doença de Chagas.

\section{Agradecimentos}

À CAPES, CNPq e FAPESP pelas bolsas concedidas e pelo apoio financeiro à pesquisa.

\section{Referências Bibliográficas}

${ }^{1}$ Moss, G. P. Pure Appl. Chem. 2000, 72, 1493. [Link] 
2 Zacchino, S.; Rodríguez, G.; Pezzenati, G.; Orellana, G. J. Nat. Prod. 1997, 60, 659. [CrossRef] [PubMed]

${ }^{3}$ Barata, L. E. S.; Santos, L. S.; Ferri, P. H.; Phillipson, J. D.; Paine, A.; Croft, S. L. Phytochemistry 2000, 55, 589. [CrossRef] [PubMed]

${ }^{4}$ Kónya, K.; Varga, Z.; Antus, S. Phytomedicine 2001, 8, 454. [CrossRef] [PubMed]

${ }^{5}$ Alves, C. N.; Barroso, L. P.; Santos, L. S.; Jardim, I. N. J. Braz. Chem. Soc. 1998, 9, 577. [CrossRef]

${ }^{6}$ Gottlieb, O. R. Revista Acta Amazon. 1988, 18, 333. [Link]

${ }^{7}$ Croteau, R.; Kutchan, T. M.; Lewis, N. G. Em Biochemistry \& Molecular Biology of Plants; Buchanan, B.; Gruissem, W.; Jones, R., eds.; American Society of Plant Physiologists: Califórnia, 2009, cap. 24. [Link] [Link2]

${ }^{8}$ Silva Filho, A. A.; Costa, E. S.; Cunha, W. R.; Silva, M. L. A.; Nanayakkara, N. P. D.; Bastos, J. K. Phytother. Res. 2008, 22, 1307. [CrossRef] [PubMed]

${ }^{9}$ Souza, V. A.; Silva, R.; Pereira, A. C.; Royo, V. A.; Saraiva, J.; Montanheiro, M.; Souza, G. H. B.; Silva Filho, A. A.; Grando, M. D.; Donate, P. M.; Bastos, J. K.; Albuquerque, S.; Silva, M. L. A. Bioorg. Med. Chem. Lett. 2005, 15, 303. [CrossRef] [PubMed]

${ }^{10}$ Silva, R.; Souza, G. H. B.; Silva, A. A.; Souza, V. A.; Pereira, A. C.; Royo, V. A.; Silva, M. L. A.; Donate, P. M.; Araújo, A. L. S. M.; Carvalho, J. C. T.; Bastos, J. K. Bioorg. Med. Chem. Lett. 2005, 15, 1033. [CrossRef] [PubMed]

${ }^{11}$ Chauret, D.; Bernard, C. B.; Arnason, J. T.; Durst, T. J. Nat. Prod. 1996, 59, 152. [CrossRef] [PubMed]

${ }^{12}$ Cow, C.; Leung, C.; Charlton, J. L. Can. J. Chem. 2000, 78, 553. [CrossRef]

13 Tanabe, Y.; Seko, S.; Nishii, Y.; Yoshida, T.; Utsumi, N.; Suzukamo, G. J. Chem. Soc. Perkin Trans. 1 1996, 2157. [CrossRef]

14 a) da Silva, F. C.; Ferreira, S. B.; da Rocha, D. R.; Ferreira, V. F. Rev. Virtual Quim. 2012, 4, 46; [Link] b) Sítio da Organização Mundial de Saúde. Disponível em:<http://www.who.int/mediacentre/factsheets/fs3 40/en/index.html>. Acesso em: 28 abril 2012.

15 a) Coura, J. R.; Castro, S. L. Mem. Inst. Oswaldo Cruz. 2002, 97, 3; [CrossRef] [PubMed] b) Coura, J. R.; Dias, J. C. P. Mem. Inst. Oswaldo Cruz. 2009 104, 31. [CrossRef] [PubMed]

${ }^{16}$ Barreto, L. M.; Texeira, M. G.; Bastos, I. F.; Ximenes, R. A. A.; Barata, R. B.; Rodrigues, L. C. Lancet 2011, 377, 1877. [CrossRef] [PubMed]
${ }^{17}$ Yadón, Z. E.; Gurtler, R. E.; Tobar, F.; Medici, A. C.; Descentralização e gestão do controle das enfermidades transmissiveis na América latina, Brasília: Organização Pan-Americana da Saúde, 2006. [Link]

${ }^{18}$ Santo, A. H. Rev. Panam. Salud. Publ. 2009, 26, 299. [CrossRef] [PubMed]

${ }^{19}$ Hoare, C. A.; Wallace, F. G. Nature 1996, 212, 1385. [CrossRef]

${ }^{20}$ Boainain, E.; Rassi, A. Arq. Bras. Cardiol. 1979, 32, 395. [Link]

21 Izumi, E.; Ueda-Nakamura, T.; Dias Filho, B. P.; Veiga Júnior, V. F.; Nakamura, C. V. Nat. Prod. Rep. 2011, 28, 809. [CrossRef] [PubMed]

22 a) Luize, P. S. L.; Ueda-Nakamura, T.; Dias Filho, B. P.; Diógenes, A. G. C.; Nakamura; C. V. Biol. Pharm. Bull. 2006, 10, 2126; [CrossRef] [PubMed] b) Pelizzaro-Rocha, K. J.; Veiga-Santos, P.; Lazarin-Bidóia, D.; Ueda-Nakamura, T.; Dias Filho, B. P.; Ximenes, V. F.; Silva, S. O.; Nakamura, C. V. Microb. Infect. 2011, 13, 1018. [CrossRef] [PubMed]

23 Bernardes, L. S. C.; Kato, M. J.; Albuquerque, S.; Carvalho, I. Bioorg. Med. Chem. 2006, 14, 7075. [CrossRef] [PubMed]

${ }^{24}$ Felippe, L. G.; Baldoqui, D. C.; Kato, M. J.; Bolzani, V. S.; Guimarães, E. F.; Cicarelli, R. M. B.; Furlan, M. Phytochemistry 2008, 69, 445. [CrossRef] [PubMed]

25 Medola, J. F.; Cintra, V. P.; Silva, E. P. P.; Royo, V. A.; Silva, R.; Saraiva, J.; Albuquerque, S.; Bastos, J. K.; Silva, M. L. A.; Tavares, D. C. Food Chem. Toxicol. 2007, 45, 638. [CrossRef] [PubMed]

${ }^{26}$ Saraiva, J.; Veja, C.; Rolon, M.; Silva, R.; Silva, M. L. A.; Donate, P. M.; Bastos, J. K.; Gomez-Bairro, A.; Albuquerque, S. Parasitol. Res. 2007, 100, 791. [CrossRef] [PubMed]

27 Landais, Y; Robin, J. P.; Lebrun, A. Tetrahedron 1991, 47, 3787. [CrossRef]

${ }^{28}$ Charlton, J. L.; Chee, G. L. Can. J. Chem. 1997, 75, 1076. [CrossRef]

${ }^{29}$ Silva, R; Saraiva, J.; Albuquerque, S.; Curti, C.; Donate, P. M.; Bianco, T. N. C.; Bastos, J. K.; Silva, M. L. A. Phytochemistry 2008, 69, 1890. [CrossRef] [PubMed]

${ }^{30}$ Del Omo, E; Armas, M. G.; Lopez-Perez, J. L.; Ruiz, G.; Vargas, F.; Gimenez, A.; Deharo, E.; Feliciano, A. S. Bioorg. Med. Chem. Lett. 2001, 11, 2755. [CrossRef] [PubMed]

${ }^{31}$ Cabral, M. M. O; Barbosa-Filho, J. M.; Maia, G. L. A.; Rev. Virtual Quim. |Vol 4| |No. 3| |197-207| 
Chaves, M. C. O.; Braga, M. V.; Souza, W.; Soares, R. O. A. Exp. Parasitol. 2010, 124, 319. [CrossRef] [PubMed]

32 Pereira, A. C.; Magalhães, L. G.; Gonçalves, U. O.; Luz, P. P.; Moraes, A. C. G.; Rodrigues, V.; Guedes, P. M. M.; Silva Filho, A. A.; Cunha, W. R.; Bastos, J. K.; Nanayakkara, N. P. D.; Silva, M. L. A. Phytochemistry 2011, 72, 1424. [CrossRef] [PubMed]
${ }^{33}$ Garcia, E. S.; Azambuja, P. Toxicon. 2004, 44, 431. [CrossRef] [PubMed]

34 Abe, F.; Nagafuji, S.; Yamauchi, T.; Okabe, H.; Maki, J.; Higo, H.; Akahane, H.; Aguilar, A.; Jiménez-Estrada, M.; Reyes-Chilpa, R. Biol. Pharm. Bull. 2002, 25, 1188. [CrossRef] [PubMed] 